Original Article (short paper)

\title{
Lack of dystrophin influences muscle inflammation but not myogenic regulatory factors after eccentric exercise in $m d x$ mice
}

\author{
Mariana Cruz Lazzarin $^{1}$ (D) , Hananiah Tardivo Quintana ${ }^{1}$ (D) , Vivianne Izabelle de Araújo Baptista ${ }^{2}$ (D) , \\ Flavia de Oliveira ${ }^{1}$
}

\author{
${ }^{1}$ Universidade Federal de São Paulo, Departamento de Biociências, Programa de \\ Pós-graduação Interdisciplinar em Ciências da Saúde, Santos, SP, Brasil; ${ }^{2}$ Universidade \\ Federal do Rio Grande do Norte, Escola Multicampi de Ciências Medicas, Caicó, RN, Brasil
}

\begin{abstract}
Aim: To investigate the consequences of chronic eccentric exercise in histopathology, inflammatory, and myogenic regulatory factors response in gastrocnemius muscle of X-chromosome-linked muscular dystrophy ( $m d x$ ) mice. Method: Male $m d x$ and control mice (C57BL/10 lineage) were distributed in the following groups: Sedentary Control (SC), Trained Control (TC), Sedentary $M d x$ (S-Mdx), and Trained $M d x$ (T-Mdx). Trained animals were subjected to downhill running for 7 weeks. Gastrocnemius was submitted to histopathological analysis and immunoexpression of Cyclooxygenase-2 (COX-2) and myogenic regulatory factors (myoD and myogenin). Results: The exercise influenced inflammation response as demonstrated by the increased COX-2 immunoexpression in T-Mdx. Interestingly, Myogenic regulatory factors revealed that the lack of dystrophin has not been influenced myoD and the increase of myogenin occurred due to exercise and was not aggravated by the absence of dystrophin. Conclusion: In conclusion, an eccentric exercise in gastrocnemius of $m d x$ mice was characterized by an intense inflammatory process without myogenic response. These findings suggest that special attention should be given to inflammatory aspects related to COX-2 associated with a decrease of myoD expression, as biomarkers in motor rehabilitation programs.
\end{abstract}

Keywords: $m d x$ mice; exercise; myoD; myogenin; COX-2.

\section{Introduction}

Duchenne muscular dystrophy (DMD) is caused by a mutation in dystrophin, a critical muscle protein that connects the cytoskeleton and the extracellular matrix ${ }^{1}$. It is a rare muscle disorder related to a deficiency in the gene responsible for coding dystrophin ${ }^{2}$. The absence of dystrophin damages skeletal muscle during contractions $\mathrm{s}^{4}$ and this lesion requires repetitive cycles of muscle fiber degeneration and regeneration.

The pathology of DMD involves muscle damage. After the injury, quiescent satellite cells are activated by inflammatory cells and up-regulate members of myogenic regulatory factors expression, myoD, and myogenin, in order to regulate myogenesis process $^{3}$. myoD stimulates differentiation of satellite cells into myogenic cells and activates myogenin, which is characterized by the promotion of the differentiation and development of myofibrils and myotubes ${ }^{4}$. Therefore, myogenin is an important modulator of cell cycle exit during differentiation, while myoD is involved in the determination of myogenic cells ${ }^{5}$. During muscle regeneration, several substances including prostaglandins are released as a product of fiber degeneration ${ }^{6}$. Prostaglandin synthesis is performed by enzymes called cyclooxygenase (COX), among them a pro-inflammatory mediator COX $-2^{7}$. After muscle injury, COX-2 expression is induced in the early stages of muscle repair to activate satellite cells myoblasts proliferation and myoD expression increases ${ }^{8}$. However, it is well established that overexpression of inflammatory markers contributes to increased fibrosis process.

In DMD disease, there are many strategies aiming to improve patients' quality of life and slow down the progression of the disease and prevent complications, considering that there is no effective therapy available for this disease. These strategies are based on induced pluripotent stem cells as a powerful technology in drug discovery ${ }^{9}$, improvement of pre-clinical models ${ }^{10}$, application of pharmacological agents that slow the progression of the disease ${ }^{11}$, and motor rehabilitation programs based on motor and functional recovery and complications prevention. According to Gaiad et al. ${ }^{12}$, motor objectives in physical therapy, such as to improve quality of life and prevent complications, are clear. In addition, Kostek ${ }^{13}$ describes motor physical therapy to focus on splinting, to prevent contractures and maintain flexibility, and non-weight bearing physical activity, such as water exercises, to provide a mild muscle stimulation. Meanwhile, Gaiad et al. ${ }^{12}$ and Kostek ${ }^{13}$ reported intense physical exercise (training) contradictions, needing further analysis due to the difficulty in identifying the ideal intensity and frequency that do not damage the muscle. Therefore, is necessary a precision exercise therapy ${ }^{13}$, which is according to the genetic variation, disease staging, and response to specific targeted therapies. To evaluate the precision exercise therapy, are important to consider a panel of biomarkers outcomes to the severity and progression of the disease and response to treatment ${ }^{14}$. 
Therefore, preclinical studies on the $m d x$ mouse model are important and could allow us to clarify the influences of the dystrophin deficiency on skeletal muscle post-exercise and biomarkers involved. In $m d x$ mice, eccentric exercise promotes sarcolemma rupture and an increased inflammatory profile of exercised skeletal muscle and accelerates regeneration/degeneration muscle cycle ${ }^{15}$. Although eccentric exercise in DMD is associated with an accelerated process of muscle degeneration, the inflammatory and myogenic regulatory factors as well as the relationship between them have not been elucidated. In this way, the muscle-injury-inducing exercise was chosen in this study to investigate, in a preclinical model of DMD, biomarkers involved in this process. Therefore, this study aimed to investigate the consequences of chronic eccentric exercise in histopathology, inflammatory, and myogenic regulatory factors response in gastrocnemius muscle of $m d x$ mice.

\section{Methods}

\section{Animals, groups and eccentric exercise protocol}

Male eight-week-old C57BL/10 lineage (control mice) and C57BL/10-Dmd ${ }^{\mathrm{mdx}}$ (a spontaneous DMD $M d x$ mutant mice that do not express dystrophin) were randomly assigned to one of the four groups $(\mathrm{n}=5)$ : Sedentary Control (SC), Trained Control (TC), Sedentary $M d x(\mathrm{~S}-M d x)$ and Trained $M d x(\mathrm{~T}-M d x)$. The animals were obtained from Fundação Oswaldo Cruz, Manguinhos - RJ (FIOCRUZ-Manguinhos), and they were kept at the laboratory animal house of the Department of Bioscience in a temperature-controlled room $\left(22^{\circ}\right)$ with a $12 \mathrm{~h}-12 \mathrm{~h}$ light-dark cycle and free access to water and food. The study was performed according to the guide proposed by the National Council for the Control of Animal Experimentation (CONCEA). All procedures were approved by the Animal Ethics Committee in the Use of Animal of the Federal University of Sao Paulo, Brazil (8165240614).

Aiming to generate the muscle-injury inducing exercise, trained groups were subjected to eccentric exercise treadmill protocol, a downhill running. The first week was used to adapt the animal to a treadmill. After adaptation week, animals were subjected to daily running on a motorized treadmill with negative inclination $\left(-15^{\circ}\right)$ at $14 \mathrm{~m} / \mathrm{min}$ for 60 minutes, for 7 weeks. The first 10 minutes of each session was used to progressively reach a speed of $14 \mathrm{~m} / \mathrm{min}$. Each session of the exercise was of high intensity for the mice. The exercise protocol was adapted by Taniguti et $\mathrm{al}^{16}$. After exercise, sixteenweek-old animals of all groups were euthanized with a lethal intraperitoneal injection of Ketamine $(150 \mathrm{mg} / \mathrm{Kg})$ and Xylazine $(30 \mathrm{mg} / \mathrm{Kg})$. Gastrocnemius muscle was dissected and fixed in $10 \%$ formalin phosphate buffer for $24 \mathrm{~h}$. The specimens were routinely embedded in paraffin blocks and cut in transversal sections $(4 \mu \mathrm{m})$. The slides were stained with hematoxylin and eosin (H\&E).

\section{Histopathological and morphoquantitative analysis}

Histopathological analysis was conducted by H\&E evaluation. In addition, a semi-quantitative assessment was performed by the muscle histopathological score (0-10) as described by Carter et al. ${ }^{17}$ The histopathology score was performed blinded by M.C. Lazzarin for each muscle group and, according to this evaluation, 0 represents a lack of histopathological alterations and 10 represents intense inflammatory infiltrate with complete tissue degeneration (Table 1):

Table 1- Histopathology scoring system

\begin{tabular}{cl}
\hline Numerical Score & \multicolumn{1}{c}{ Skeletal Muscle Histopathology } \\
\hline 0 & No abnormal findings \\
1 & Mild localized mononuclear cell infiltration \\
2 & Mild multifocal mononuclear cell infiltration, rare necrotic fibers \\
3 & Moderate generalized mononuclear cell infiltrations, occasional necrotic fibers \\
4 & Moderate cell infiltration including PMN, mild multifocal necrosis \\
5 & Marked cell infiltration including PMN, moderate multifocal fiber necrosis \\
6 & Marked cell infiltration including PMN, moderate generalized fiber necrosis \\
7 & Severe cell infiltration including PMN, marked multifocal fiber necrosis \\
8 & Severe cell infiltration, hemorrhage possible with severe fiber necrosis \\
9 & Massive cell infiltration, hemorrhage possible with severe generalized fiber necrosis \\
10 & Massive cell infiltration, and complete loss of tissue architecture \\
\hline
\end{tabular}

PMN, polymorphonuclear cells.

Carter et al., ${ }^{20}$

Motriz, Rio Claro, v.26, Issue 3, 2020, e10200228 
Morphometric analysis was investigated by muscle fibers cross-sectional area, as performed by Shavlakadze et al. ${ }^{18}$, Timpani et al. ${ }^{19}$ and Timpani et al..$^{20}$, using $m d x$ animals. For this purpose, one cut from all six sections stained with $\mathrm{H} \& \mathrm{E}$ for each animal was chosen randomly. Five fields from each section chosen were photographed with a 40x objective lens (Axio Observer.D1, Zeiss ${ }^{\circledR}$, Thornwood, NY, USA). To measure the area of muscle fibers, a delimited area of $10,000 \mu \mathrm{m}^{2}$ standardized by a test frame was considered ${ }^{21}$. All cells located within this delimited area were measured and the area values were obtained in $\mu \mathrm{m}^{2}$. The distribution frequency of the muscle fibers cross-sectional area was presented in a histogram.

\section{Immunohistochemical analysis: Myogenic regulatory factors (myoD, myogenin) and inflammation (COX-2)}

The paraffin of serial muscle sections $(4 \mu \mathrm{m})$ was removed with xylene and sections were rehydrated in graded ethanol and then pre-treated with $0.01 \mathrm{M}$ citric acid buffer $(\mathrm{pH} 6)$ in a microwave for $15 \mathrm{~min}$ at $850 \mathrm{~W}$ for antigen retrieval. The sections were pre-incubated for $5 \mathrm{~min}$ in $0.3 \%$ hydrogen peroxide in phosphate-buffered saline (PBS) solution to inactive the endogenous peroxidase. Then the material was blocked with $5 \%$ normal goat serum in PBS solution for $10 \mathrm{~min}$. Specimens were incubated with primary antibodies: anti-MyoD (dilution 1:100, clone 5.8A, sc-32758, Santa Cruz Biotechnology), anti-myogenin (dilution 1:100, clone 5FD, sc-52903, Santa Cruz Biotechnology), and anti-COX-2 (dilution 1:100, clone M19, sc-1747, Santa Cruz Biotechnology). Sections were incubated overnight at $4^{\circ} \mathrm{C}$ in a refrigerator. Then they were washed in PBS and incubated with biotin-conjugated secondary antibody anti-rabbit IgG (Vector Laboratories, Burlingame, CA) at a dilution of 1:200 in PBS for $30 \mathrm{~min}$, washed with PBS, followed by the application of performed avidin-biotin complex conjugated to peroxidase (Vector Laboratories) for $30 \mathrm{~min}$. Then continued with the application of a $0.05 \%$ solution of a 3-3-diaminobenzidine solution and counterstained with Harris hematoxylin (Merck). Negative controls were analyzed by omitting the primary antibody from the staining procedure. Additionally, internal positive controls were performed with each staining bath.

Both, myoD and myogenin were investigated by analyzing immunoreactive nuclei density ( $\%$ immunoreactive nuclei). The $\%$ immunoreactive nuclei were calculated from five random photomicrographs per animal and were carried out using a computerized imaging system (software Axio Visio 4.5 Zeiss) with an objective magnification of $x 40$. The number of myoD and myogenin immunoreactive nuclei were counted by an evaluator and expressed as a percentage relative to the number of total nuclei ${ }^{21,22}$. Cytoplasmatic immunoexpression (COX-2) was established to determine the percentage of the immunoreactive area (\% immunoreactive area), calculated from five random photomicrographs per animal, and were carried out using a computerized imaging system (software Axio Visio 4.5 Zeiss) with the objective magnification of $x 40$. The percentage of immunoreactive COX-2 area was quantified as a COX-2-immunomarked percentage relative to the total image field taken ${ }^{23}$, using the mentioned software.

\section{Statistical treatment}

All data are expressed as mean + standard deviation (SD). Gastrocnemius muscle was evaluated by analysis of variance (ANOVA) with two factors (group and exercise), and followed with a Tukey's test for multiple comparisons, when necessary. $\mathrm{P}<0.05$ was considered to be statistically significant. All analysis was performed using the R Core Team software (R Core Team, Vienna, Austria).

\section{Results}

\section{Effects of exercise on histopathological and morphoquantitative parameters}

$C$ groups (SC and TC) sections for gastrocnemius muscle exhibited fibers with peripheral nuclei, a polygonal shape, and homogeneous morphology. In contrast, $M d x$ groups (S- $M d x$ and $\mathrm{T}-M d x$ ) showed a wide variety of morphology (polygonal and round muscle fibers), different sizes, centralized and peripheral nuclei, fiber necrosis, and inflammatory infiltrate (Figure 1).

Histopathological score revealed that $M d x$ groups had higher scores than $\mathrm{C}$ groups $(\mathrm{p}<0.05)$. Eccentric exercise training did not influence the histopathology of the gastrocnemius (Figure 2A).

$M d x$ groups showed muscle fiber cross-sectional area values between 50 and $4.300 \mu \mathrm{m}^{2}$ and Control groups had a lower numerical range between 200 and $2.100 \mu \mathrm{m}^{2}$. A comparative analysis between groups revealed that control groups presented two frequency peaks: $500-700 \mu \mathrm{m}^{2}$ and $1.100-1.300 \mu \mathrm{m}^{2}$. In contrast, the $M d x$ groups showed only one peak of distribution frequency between $200-400 \mu \mathrm{m}^{2}$. Note that T- $M d x$ presented higher frequency (27\%) on peak than S-Mdx, with $20 \%$ (Figure 2B).

\section{Effects of exercise on myogenic regulatory factors (myoD and myogenin) and inflammatory response $(C O X-2)$}

Both, myoD and myogenin immunoexpression were detected in muscle fiber nuclei. Gastrocnemius of $M d x$, in general, had a lower percentage of myoD immunoreactive nuclei than Control groups $(p<0.05)$ and eccentric exercise did not cause differences among the groups. Concerning myogenin, $M d x$ groups (S- $M d x$ and T-Mdx) had a lower percentage of immunoreactive nuclei than $\mathrm{C}$ groups. However, it was observed that eccentric exercise was able to increase the percentage of immunoreactive nuclei in the $\mathrm{C}$ group $(\mathrm{TC}>\mathrm{SC})$ and $M d x$ group (T-Mdx $>\mathrm{S}-M d x$ ) (Figure 3-4).

COX-2 immunoexpression in gastrocnemius evidenced higher immunoreactivity area in $M d x$ groups when compared to $\mathrm{C}$ groups $(\mathrm{p}<0.05)$. Regarding the eccentric exercise effect, it was verified that the exercise exacerbated the inflammatory response investigated by COX-2 $(\mathrm{p}<0.05)$ (Figure 5-6). 


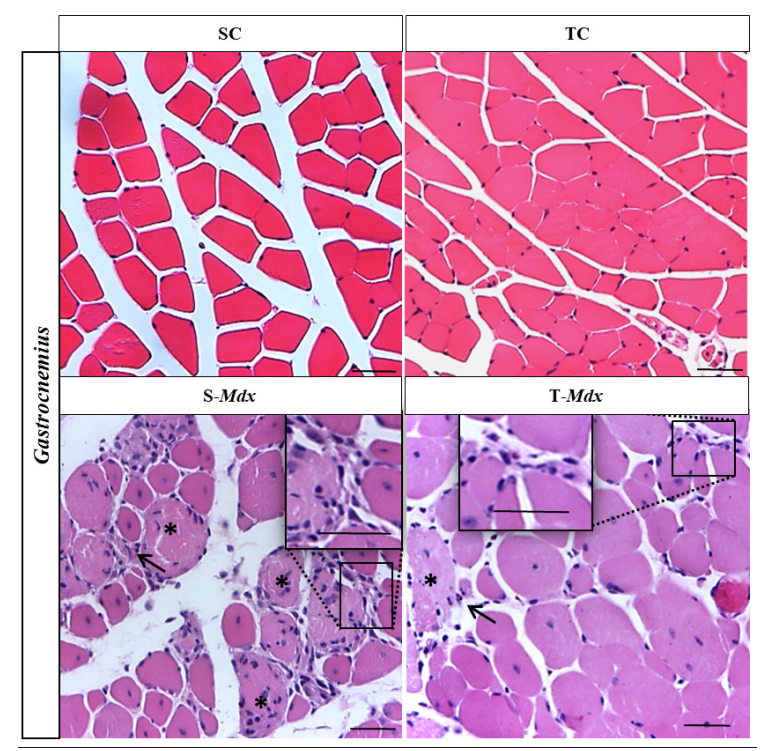

Figure - 1 Effects of exercise on gastrocnemius muscle of mice stained with H\&E. SC and TC groups presented fibers with equidistant space, homogeneous size and peripheral nuclei. $\mathrm{S}-\mathrm{Mdx}$ arrows and prominence image indicate intense inflammatory infiltration. Asterisk shows rounded cells undergoing degeneration process. Thin arrows and prominence image in T-Mdx indicate cells with heterogeneous size, centralized nuclei, moderate inflammatory infiltration, and asterisk demonstrates cells in necrosis. Bar $=50 \mu \mathrm{m}$.

$\mathbf{A}$

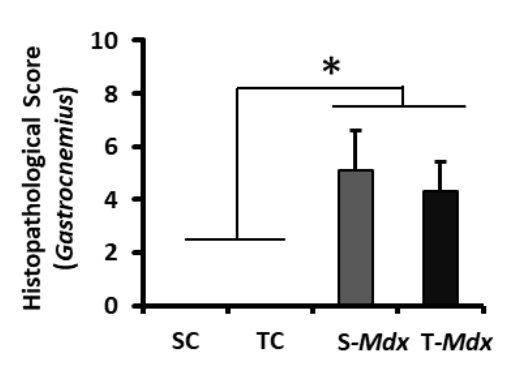

B

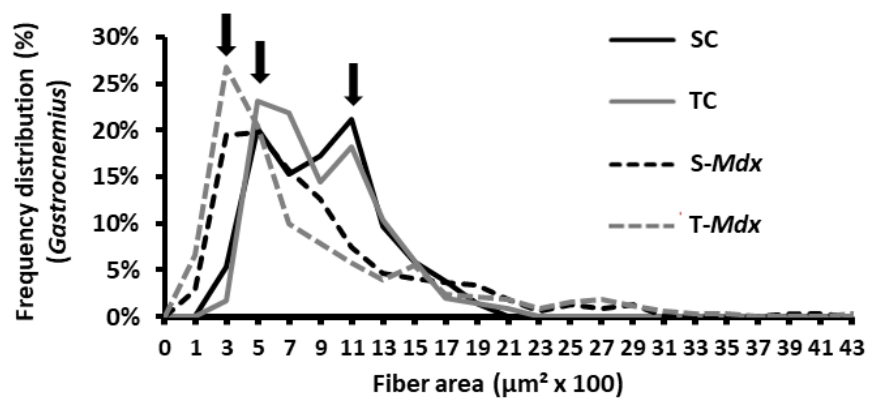

Figure - 2 Histopathological score (A) and muscle fiber cross sectional area frequency distribution (B). The graph (A) shows gastrocnemius histopathological score (Mean + S.D.) of Mdx groups were higher than $\mathrm{C}$ groups $(*-p<0.05)$. The graph $(\mathrm{B})$ shows muscle fiber area frequency distribution of gastrocnemius muscle and arrows show peaks of frequency.

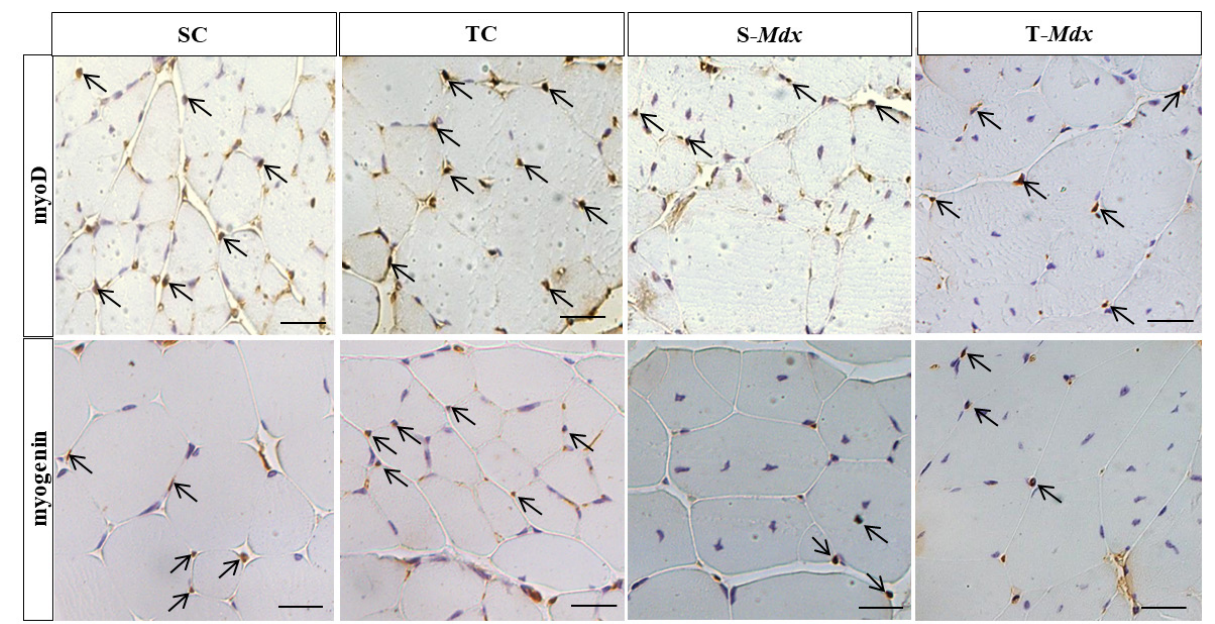

Figure - 3 Effects of exercise on both myoD and myogenin immunoexpression of gastrocnemius muscle. Thin arrows highlight nuclear immunoexpression. Bar $=50 \mu \mathrm{m}$. 

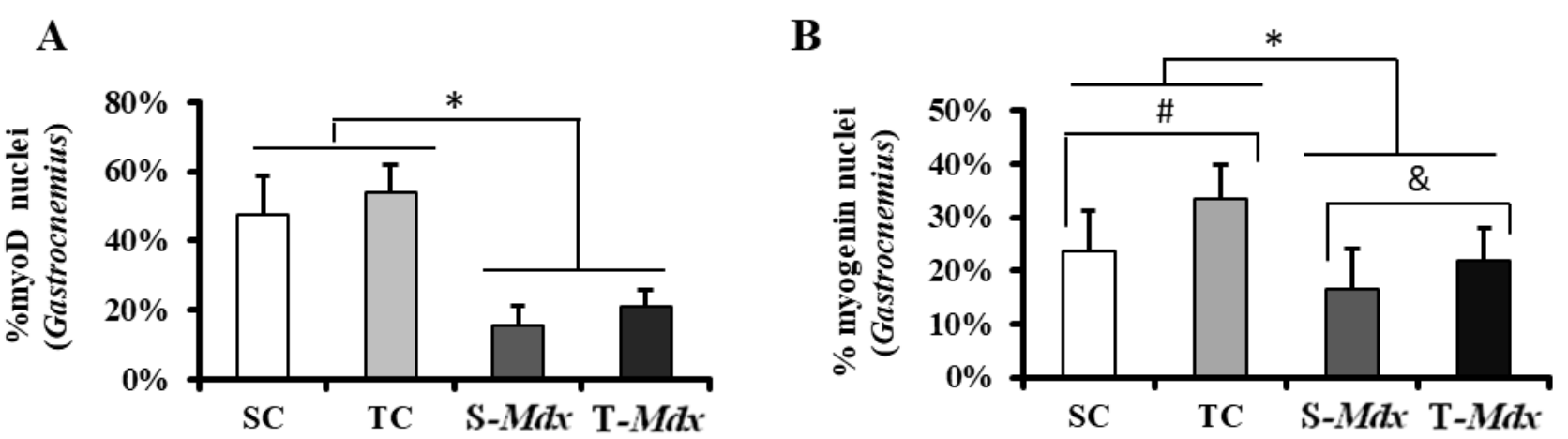

Figure - 4 Both, myoD (A) and myogenin (B) graphs show the percentage of immunoreactive nuclei (Mean + S.D.). In (A), the percentage of myoD immunoreactive nuclei in Mdx groups were lower than $\mathrm{C}$ groups $\left({ }^{*}=\mathrm{p}<0.05\right)$. In $(\mathrm{B})$, the percentage of myogenin immunoreactive nuclei in Mdx groups were lower than $\mathrm{C}$ groups $(*=\mathrm{p}<0.05)$. In addition, TC was higher than SC group $(\#=\mathrm{p}<0.05)$ and T-Mdx was higher than $\mathrm{S}-\mathrm{Mdx}$ group $(\&=\mathrm{p}<0.05)$.

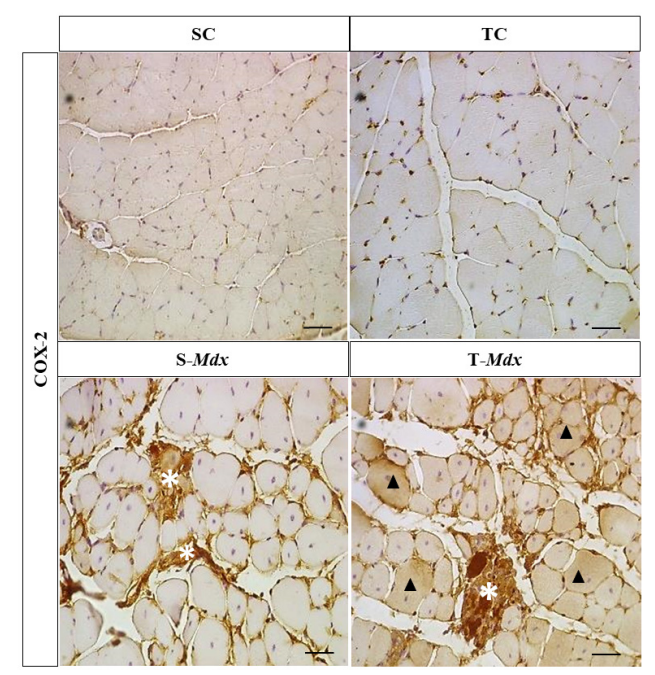

Figure - 5 Effects of exercise on COX-2 immunoexpression. Asterisks in S-Mdx and T-Mdx shows connective tissue thickening in infiltrate inflammatiory areas.

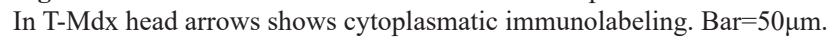

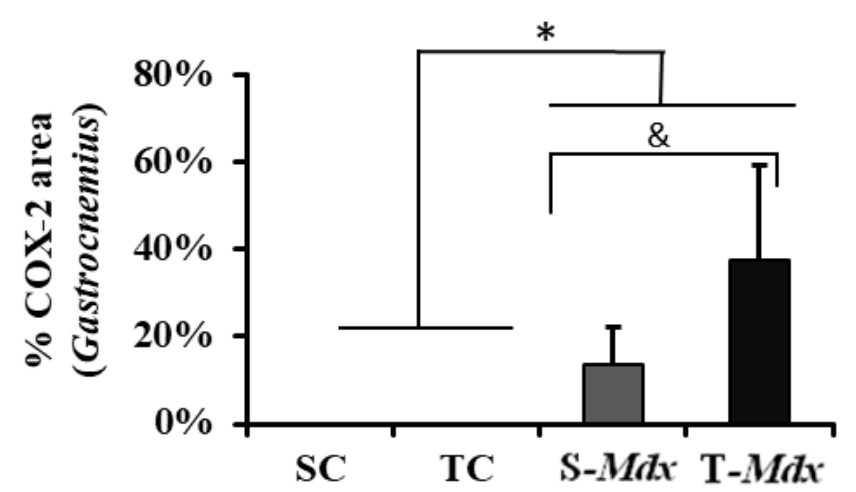

Figure - 6 Graph shows the percentage of COX-2 immunoreactive area (Mean + S.D) higher in Mdx groups than in C groups $(*=p<0.05)$; and T-Mdx higher than $\mathrm{S}-\mathrm{Mdx}$ group $(\&=\mathrm{p}<0.05)$. 


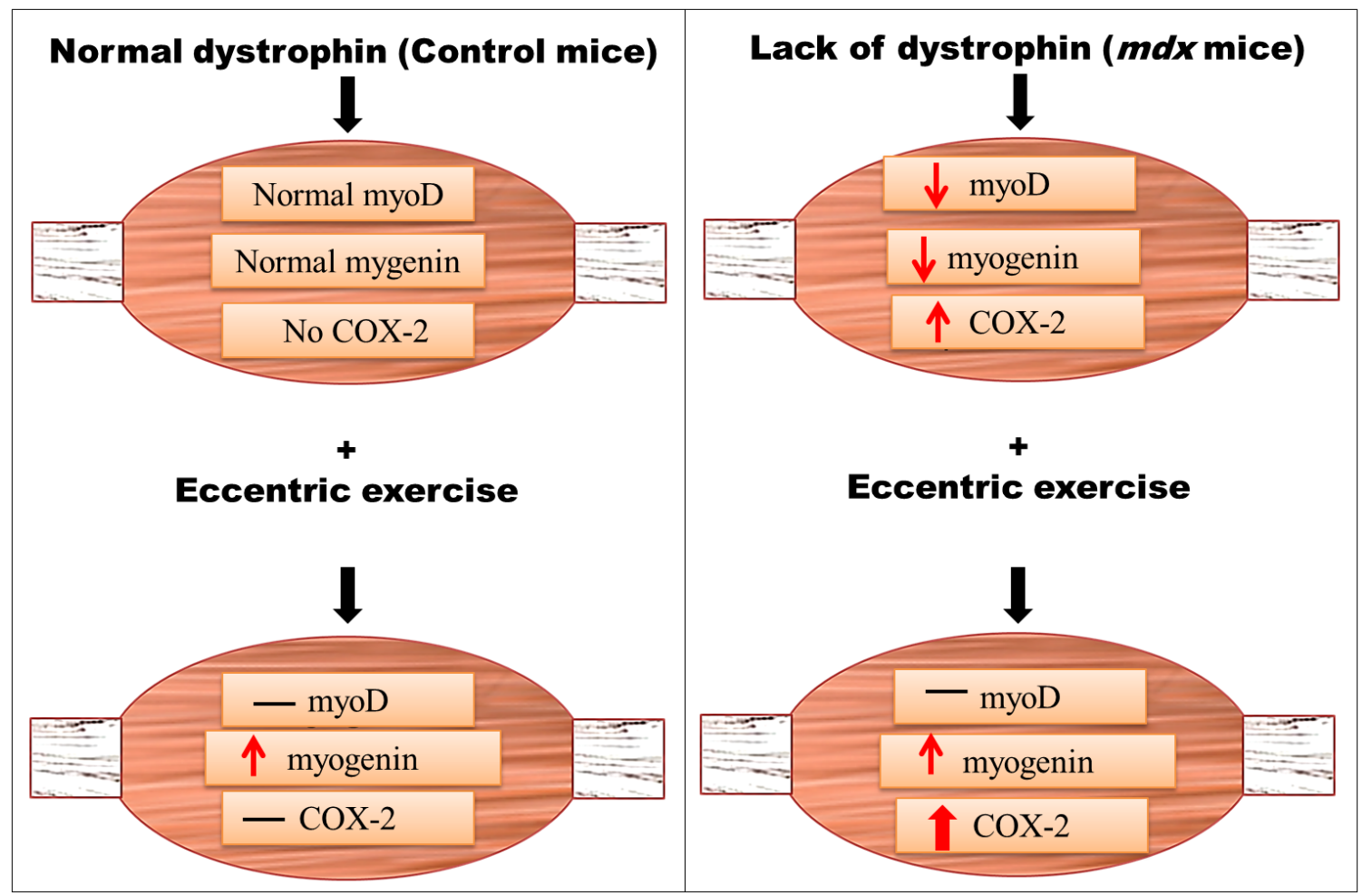

Figure - 7 Schematic response of biomarkers in Control (normal dystrophin) and Mdx (lack of dystrophin) mice after eccentric exercise training. After training, Control mice increase myogenin and do not alters myoD and COX-2 immunoexpressions. Regarding mdx mice, lack of dystrophin provokes decrease of myogenic regulatory factors (myoD and myogenin) and increase of COX-2. After eccentric exercise training, myoD immunoexpression in mdx do not alter but myogenin increase and COX-2 had exacerbated response. Note that the increase of myogenin occurred due to exercise and not aggravated by lack of dystrophin.

\section{Discussion}

The relationship between a panel of biomarkers and exercise outcomes is necessary to establish an indication of disease progression and motor treatment response. Therefore, this study of the pre-clinical $m d x$ mouse model allows clarifying the influence of the dystrophin deficiency on skeletal muscle after exercise as well as biomarkers involved, considering that a chronic eccentric exercise program associated with the accelerated process of muscle degeneration was chosen. The main results revealed that eccentric exercise provokes exacerbated inflammatory response by COX-2 increase in $M d x$ trained group, but, at the same time, the response of myogenic regulatory factors (myoD and myogenin) revealed that the lack of dystrophin has not been influenced myoD and the increase of myogenin occurred due to exercise and was not aggravated by the absence of dystrophin (Figure 7).

The muscle researched suffered the direct influence of physical exercise, because gastrocnemius, responsible for animal locomotion has been widely recruited in treadmill protocols. In addition, it has been well established that gastrocnemius muscle is composed of a mix of fiber types, representing one good model for muscle morphological and functional features. Downhill running is an eccentrically biased exercise used as a tool to generate muscle damage. In muscle of rodents, downhill running promotes muscle membrane rupture and elevations in plasma $\mathrm{CK}$, local inflammation with aggregations of leukocytes, and regeneration with satellite cell activation ${ }^{24}$. However, in the $m d x$ mice, whose regeneration capacity is compromised, chronic downhill running protocol increase muscle fibrosis and decrease forelimb strength ${ }^{25} . M d x$ mice showed typical histopathological alterations ${ }^{26}$ with rounded cell and centralized nuclei, heterogenic sizes of fibers, and big spaces between muscle fascicles. In addition, results revealed high histopathological scores obtained by the presence of cells in the degeneration process or necrosis and inflammatory infiltrate. Despite the large differences between control groups and $M d x$ groups in histopathological findings, interestingly eccentric exercise did not aggravate the $M d x$ group because no statistical difference was found between sedentary and trained mice.

In our investigation, different degrees of involvement were introduced in the analysis of the muscle fibers cross-sectional area, and data collected corresponded to a wide variation in fiber size and necrotic fibers presence accompanied by inflammatory infiltrate. These characteristics contributed to the high heterogeneity of muscle fibers cross sectional area in $M d x$ mice. According to Duddy et al. ${ }^{27}$, the heterogeneity of skeletal muscle fibers of $m d x$ mice is composed of hypotrophy, hypertrophy, and hyperplasia processes. Because of the heterogeneity of muscle fibers cross-sectional area in $M d x$, this study analyzed the range of fibers area and frequency peaks. Gastrocnemius fibers of $m d x$ mice had a higher percentage of cells with reduced size associated with a very wide range of 
cell size (up to $4.300 \mu \mathrm{m}^{2}$ ), while the control group presented cell size up to $2.100 \mu \mathrm{m}^{2}$, in general. A large percentage of cells with reduced size identified in this study possibly corresponds to the increased number of myonuclei per muscle fibers in growth for regeneration attempt. Conversely, the larger size of some fibers found in $M d x$ groups is in line with the wide variety of round fibers identified in the histopathology. Notably, T-Mdx presented a higher percentage of small fibers than S-Mdx. These muscle fibers with a small area can be represented by myoblast proliferation stimulated by exercise.

The morphometric changes found in dystrophic muscle and the effect of exercise may be related to muscle regeneration mechanisms, as the expression of myogenic regulatory factors, myoD, and myogenin. Following injury, satellite cells are activated and differentiate into myoblasts, stimulated by myogenic regulatory factor myoD. The myoblasts upregulate the expression of myogenin, promoting to the exit of the cell cycle, maturation, and fusion of myoblasts to form newly regenerating myofibers ${ }^{28}$. Therefore, myoD expression is essential to satellite cell proliferation and differentiation into myoblasts, while the myogenin is expressed during myoblast formation, stimulated maturation, and generating the muscle fiber ${ }^{6}$. Our data showed reduced regenerative capacity in $m d x$ mice, with low values for myoD and myogenin when compared with control groups. This indicates that differentiation and proliferation of satellite cells, as well as the maturation of myotubules, were injured by muscular dystrophy. Gastrocnemius $m d x$ animals had lower myoD immunoreactive nuclei than the Control group. In this study, muscle $m d x$ mice have not been influenced by exercise probably because of the age of the animals evaluated (considered young). In fact, according to Dellorusso et al. ${ }^{29}$, muscles of $m d x$ mice appear to experience increased susceptibility to contraction-induced injury with age.

The myogenin expression in gastrocnemius showed lower values in $m d x$ animals than in the control groups. However, eccentric exercise promoted increased myogenin immunoreactivity in $m d x$ and Control animals after eccentric exercise (TC $>$ SC and T-Mdx>S-Mdx). Similar results about the effects of exercise were observed by Siu et al. ${ }^{30}$, which reported increased expression of myogenin after physical training in rats. As expected, myogenin expression has been increased in T-Mdx due to activated repair in the latter stages of differentiation as a result of muscle hypertrophy characterized by higher fiber calibers stimulated by exercise. However, interestingly this increment of myogenin after eccentric exercise in dystrophic mice occurred in the control group as well because myogenin of trained control mice was higher than sedentary mice. In a study of the role of myogenin in $m d x$ mice, Meadows et al. ${ }^{31}$ concluded that although myogenin expression is elevated in $M d x$ mice, myogenin is not required for survival or progression of muscular dystrophy. Therefore, in this study, we suggest that the increase of myogenin occurred due to exercise and was not aggravated by a lack of dystrophin.

To understand the role of the inflammatory response caused by eccentric exercise in $m d x$ mice, we investigated the COX-2 immunoexpression on gastrocnemius muscle. Several studies have demonstrated that COX-2 plays a crucial role after skeletal muscle injury using different experimental models ${ }^{8,32}$. Nevertheless, studies investigating COX-2 immunoexpression in $m d x$ muscle mice as a consequence of a chronic excentric program response were not performed so far COX-2 plays a crucial role in the initial process of muscle regeneration, activating satellite cells and myoblasts proliferation of increased myoD expression ${ }^{8}$. In this study, greater COX-2 immunoexpression in $M d x$ groups was observed in the same space occupied by the altered conjunctive tissue corresponding to necrotic and fibrotic lesions. This characterizes a chronic state of inflammation in dystrophic muscle, as described by Martinez et al. ${ }^{33}$, who detected the progress of the inflammatory process related to $\mathrm{COX}-2$ expression in mice dystrophic muscle. T- $M d x$ showed greater COX-2 immunoexpression when compared with the S-Mdx group. Increased COX-2 immunoexpression in dystrophic muscles is due to eccentric exercise that promotes mechanical disruption of the sarcolemma, increasing the local inflammatory process to achieve tissue regeneration. In addition, chronic inflammation in dystrophic muscle analyzed histologically and by COX-2 immunoexpression, stimulates pro-fibrotic factors, increasing extracellular matrix, and stimulating fibrotic process ${ }^{34}$. In this study, gastrocnemius suffers exacerbated inflammation effect of eccentric exercise because due to a lack of dystrophin, leading to an undesirable increment of inflammatory muscle profile.

\section{Conclusion}

In conclusion, an eccentric exercise in gastrocnemius of $m d x$ mice was characterized for an intense inflammatory process without myogenic response, since myoD was not altered and the increase of myogenin occurred due to exercise and was not aggravated by lack of dystrophin. According to the data collected, the undesirable effects of exercise in skeletal muscle of DMD disease are related mainly to the inflammation process resultant from a decreased capacity of regeneration, and the expression of COX-2 is associated with a decrease of myoD expression serves as an indicator for motor programs of rehabilitation exercise. There are very few studies that examined exercise in DMD patients or experimental models, and COX-2 response in limb reinforces the understanding, as it is well established in the literature, that extensive exercise can represent a serious disadvantage in DMD. Future studies are necessary to better understand less invasive methods of monitoring exercise (e.g. the levels of inflammatory mediators and myogenic regulatory factors response in blood and urine) in DMD patients on therapy and during day-to-day activities, with aiming to maintain the maximum muscular integrity possible as the disease progresses and in response to motor rehabilitation programs.

\section{References}

1. Hoffman EP, Brown RH Jr, Kunkel LM. Dystrophin: the protein product of the Duchenne muscular dystrophy locus. Cell. 1987;51(6):919-28. DOI:10.1016/0092-8674(87)90579-4. 
2. Mendell JR, Shilling C, Leslie ND, Flanigan KM, al-Dahhak R, Gastier-Foster J, et al. Evidence-based path to newborn screening for Duchenne muscular dystrophy. Ann Neurol. 2012;71(3):30413. DOI:10.1002/ana.23528.

3. Shi X, Garry DJ. Muscle stem cells in development, regeneration, and disease. Genes Dev. 2006;20(13):1692-708.doi: 10.1101/gad.1419406.

4. Megeney LA, Kablar B, Garrett K, Anderson JE, Rudnicki MA. MyoD is required for myogenic stem cell function in adult skeletal muscle. Genes Dev. 1996;10(10):1173-83. DOI:10.1101/gad.10.10.1173.

5. Singh K, Dilworth FJ. Differential modulation of cell cycle progression distinguishes members of the myogenic regulatory factor family of transcription factors. FEBS J. 2013;280(17):3991-4003. DOI: 10.1111/febs.12188.

6. Prisk V, Huard J. Muscle injuries and repair: the role of prostaglandins and inflammation. HistolHistopathol. 2003;18(4):1243-56. DOI:10.14670/HH-18.1243.

7. Gilroy DW, Colville-Nash PR, Willis D, Chivers J, Paul-Clark MJ, Willoughby DA. Inducible cyclooxygenase may have anti-inflammatory properties. Nat Med. 1999;5(6):698-701. DOI: $10.1038 / 9550$.

8. Bondesen BA, Mills ST, Kegley KM, Pavlath GK. The COX-2 pathway is essential during the early stages of skeletal muscle regeneration. Am J Physiol Cell Physiol. 2004;287(2):C47583. DOI: 10.1152/ajpcell.00088.2004.

9. Danisovic L, Culenova M, Csobonyeiova M. Induced Pluripotent Stem Cells for Duchenne Muscular Dystrophy Modeling and Therapy. Cells. 2018; 7(12):253. DOI: 10.3390/ cells 7120253 .

10. Gordish-Dressman H, Willmann R, DallePazze L, Kreibich A, van Putten M, Heydemann A, et al. "Of Mice and Measures": A Project to Improve How We Advance Duchenne Muscular Dystrophy Therapies to the Clinic. J Neuromuscul Dis. 2018;5(4):407-417. DOI: 10.3233/JND-180324.

11. Guiraud S, Davies KE. Pharmacological advances for treatment in Duchenne muscular dystrophy. CurrOpinPharmacol. 2017;34:36-48. DOI:0.1016/j.coph.2017.04.002.

12. Gaiad TP, Araujo KP, Serrão JC, Miglino MA, Ambrósio CE. Motor physical therapy affects muscle collagen type I and decreases gait speed in dystrophin-deficient dogs. PLoS One.2014;9(4):e93500. DOI: 10.1371/journal.pone.0093500.

13. Kostek M. Precision Medicine and Exercise Therapy in Duchenne Muscular Dystrophy. Sports. 2019;7(3):64. DOI: 10.3390 /sports 7030064 .

14. Szigyarto CA, Spitali P. Biomarkers of Duchenne muscular dystrophy: current findings. DegenerNeurolNeuromuscul Dis. 2018;8:1-13. DOI:10.2147/DNND.S121099.

15. McNeil PL, Khakee R. Disruptions of muscle fiber plasma membranes. Role in exercise-induced damage. Am J Pathol. 1992;140(5):1097-109.

16. Taniguti, APT, Pertille, A, Matsumura, CY, Neto, HS, Marques, MJ. Prevention of muscle fibrosis and myonecrosis in mdx mice by suramin, a TGF $\square \beta 1$ blocker. Muscle Nerve. 2011;43(1):82-87. DOI:10.1002/mus.21869.

17. Carter WO, Bull C, Bortolon E, Yang L, Jesmok GJ, Gundel RH. A murine skeletal muscle ischemia-reperfusion injury model: differential pathology in BALB/c and DBA/2N mice. J ApplPhysiol (1985).1998;85(5):1676-83.doi:10.1152/jappl.1998.85.5.1676.

18. Shavlakadze T, White J, Hoh JF, Rosenthal N, Grounds MD. Targeted expression of insulin-like growth factor-I reduces early myofiber necrosis in dystrophic MDX mice. Mol Ther. 2004;10(5):829-843. DOI:10.1016/j.ymthe.2004.07.026.

19. Timpani CA, Trewin AJ, Stojanovska V, Robinson A, Goodman CA, Nurgali K, et al. Attempting to Compensate for Reduced Neuronal Nitric Oxide Synthase Protein with Nitrate Supplementation Cannot Overcome Metabolic Dysfunction but Rather Has Detrimental Effects in Dystrophin-Deficient MDX Muscle. Neurotherapeutics. 2017;14(2):429-446. DOI:10.1007/s13311-016-0494-7.

20. Timpani CA, Goodman CA, Stathis CG, White JD, Mamchaoui K, Butler-Browne G, et al. Adenylosuccinic acid therapy ameliorates murine Duchenne Muscular Dystrophy. Sci Rep. 2020;10(1):1125. DOI:10.1038/ s41598-020-57610-w.

21. Quintana HT, Bortolin JA, da Silva NT, Ribeiro FAP, Liberti EA, Ribeiro DA, et al. Temporal study following burn injury in young rats is associated with skeletal muscle atrophy, inflammation, and altered myogenic regulatory factors. Inflamm Res. 2015;64(1):53-62. DOI: 10.1007/s00011-014-0783-8.

22. Sin TK, Pei XM, Teng BT, Tam EW, Yung BY, Siu PM. Oxidative stress and DNA damage signaling in skeletal muscle in pressure-induced deep tissue injury. Pflugers Arch. 2013;465(2):295-317. DOI:10.1007/s00424-012-1205-9

23. Sinicrope FA, Hart J, Michelassi F, Lee JJ. Prognostic value of BCL-2 oncoprotein expression in stage II colon carcinoma. Clin Cancer Res. 1995;1(10):1103-1110.

24. Paulsen G, Ramer Mikkelsen U, Raastad T, Peake JM. Leucocytes, cytokines, and satellite cells: what role do they play in muscle damage and regeneration following eccentric exercise? Exercise immunology review. 2012;18:42-97.

25. Spaulding HR, Selsby JT. Is exercise the right medicine for dystrophic muscle? Med. Sci. Sports Exerc. 2018;50(9):17231732. DOI: $10.1249 / \mathrm{MSS} .0000000000001639$.

26. Coulton GR, Morgan JE, Partridge TA, Sloper JC. The mdx mouse skeletal muscle myopathy: I. A histological, morphometric, and biochemical investigation. Neuropathol Appl Neurobiol. 1988;14(1):53-70.

27. Duddy W, Duguez S, Johnston H, Cohen TV, Phadke A, Gordish-Dressman H, et al. Muscular dystrophy in the $\mathrm{mdx}$ mouse is a severe myopathy compounded by hypotrophy, hypertrophy, and hyperplasia. Skelet Muscle. 2016;5(1):16. DOI: 10.1186/s13395-015-0041-y.

28. Dumont NA, Wang YX, Rudnicki MA. Intrinsic and extrinsic mechanisms regulating satellite cell function. Development. 2016;142(9):1572-81.doi:10.1242/dev.114223.

29. Dellorusso C, Crawford RW, Chamberlain JS, Brooks SV. Tibialis anterior muscles in $\mathrm{mdx}$ mice are highly susceptible to contraction-induced injury. J Muscle Res Cell Motil. 2001;22(5):467-75.

30. Siu PM, Donley DA, Bryner RW, Alway SE. Myogenin and oxidative enzyme gene expression levels are elevated in rat soleus muscles after endurance training. J ApplPhysiol(1985). 2004;97(1):27785. DOI: 10.1152/japplphysiol.00534.2004.

31. Meadows E, Flynn JM, Klein WH. Myogenin regulates exercise capacity but is dispensable for skeletal muscle regeneration in adult 
mdx mice. PLoS One. 2011;6(1):e16184. DOI:10.1371/journal. pone. 0016184 .

32. Da Silva NT, Quintana HT, Bortolin JA, Ribeiro DA, de Oliveira F. Burn injury induces skeletal muscle degeneration, inflammatory host response, and oxidative stress in Wistar rats. J Burn Care Res. 2015;36(3):428-33. DOI:10.1097/BCR.0000000000000122.

33. Martinez L, Ermolova NV, Ishikawa TO, Stout DB, Herschman HR, Spencer MJ. A reporter mouse for optical imaging of inflammation in MDX muscles. Skelet Muscle. 2015;5(1):15. DOI: 10.1186/ s13395-015-0042-x.

34. Mann CJ, Perdiguero E, Kharraz Y, guilar S, Pessina P, Serrano AL, et al. Aberrant repair and fibrosis development in skeletal muscle. Skelet Muscle. 2011;(1)1:21. DOI: 10.1186/2044-5040-1-21.

\section{Acknowledgements}

This study was supported by Fundação de Amparo à Pesquisa do Estado de São Paulo (FAPESP), process number 2014/22211-6.

\section{Corresponding author}

Flavia de Oliveira

Federal University of São Paulo, Department of Biosciences. Rua Silva Jardim, 136 - Lab 328, 11015-020, Santos/SP. Brazil. Telephone: +55 (13) 3878-3844

Email: flavia.oliveira@unifesp.br

Associate Editor: Fernanda B. M. Priviero. Augusta University, US.

Manuscript received on December 19, 2019

Manuscript accepted on August 22, 2020

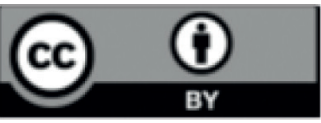

Motriz. The Journal of Physical Education. UNESP. Rio Claro, SP, Brazil - eISSN: 1980-6574 - under a license Creative Commons - Version 4.0 